\title{
The use of three-dimensional models in tibial plateau fractures
}

\author{
Joubert JA (B)
}

MBChB, FC Orth(SA), MMed(Orth); Orthopaedic Surgeon, Department of Orthopaedics, Sefako Makgatho Health Sciences University, Ga-Rankuwa, South Africa

Corresponding author: Dr JA Joubert, Life Mercantile Hospital, Room 306, Korsten, Port Elizabeth, 8020; tel: 0871511811; email: drjajoubert@gmail.com

\section{Abstract}

Background: To compare three-dimensional (3D) printed models with 3D computed tomography (CT) images in terms of the reliability of the classification of tibial plateau fractures using the Hohl and Moore and the Schatzker classification systems, and whether there was any influence on surgical management.

Method: The sample comprised the 20 CT scans of patients with tibial plateau fractures available in the radiology archive of Dr George Mukhari Academic Hospital (Ga-Rankuwa). Standardised illustrated guides to the Schatzker and the Hohl and Moore classification systems were used by six observers of varying orthopaedic experience. They completed questionnaires individually in three different sessions. In session one the observers had access to the 3D CT images only. On average two to five days later, in session two the observers assessed only the 3D models. In the third session, another two-to-five days later, the observers assessed the 3D CT images while handling and inspecting the corresponding 3D model.

Results: The inter-observer reliability of the Schatzker system (moderate reliability) was superior to the Hohl and Moore system (fair reliability) when comparing the assessment using the images and models separately. When all observations were combined, there was no difference between the systems. For the six possible surgical management options given to the observers, there was an overall difference in agreement based on assessing only the CT images compared with assessing only the models, of $19 \%$ ( 23 of the possible 120 procedures). Of these 23 differing procedures, 15 were more invasive. The 3D models were considered superior to 3D CT imaging in terms of spatial awareness and the observers' ability to assess intra-articular fracture patterns. The models were assessed as being superior to the imaging when considering the ability to estimate the quantity of bone graft required.

Conclusion: Although the inter-rater reliability of classification was not significantly improved, there were several advantages to using the 3D-printed models both separately from the CT images and together with the images. The effect of 3D models on patient outcome remains untested. The clinical impact of the use of 3D models (including cost, manufacturing time and radiation exposure) should be weighed against the potential benefits.

\section{Level of evidence: Level 4}

Keywords: tibial plateau fracture classification, 3D CT images, 3D-printed models

Citation: Joubert JA. The use of three-dimensional models to improve the reliability of tibial plateau fracture classification and their influence on surgical management. SA Orthop J 2020;19(2):79-83. http://dx.doi.org/10.17159/2309-8309/2020/v19n2a4

Editor: Prof. Nando Ferreira, Stellenbosch University, South Africa

Received: July 2019

Accepted: November 2019

Published: May 2020

Copyright: ( 2020 Joubert JA. This is an open-access article distributed under the terms of the Creative Commons Attribution Licence, which permits unrestricted use, distribution and reproduction in any medium, provided the original author and source are credited.

Funding: No funding was received for the purposes of performing this research.

Conflict of interest: The author has no conflicting interests with respect to this article. 


\section{Introduction}

One of the objectives of orthopaedic surgery is to assess and classify fractures as a standardised methodical approach, specific to each fracture site, that directs patient treatment and warns against certain complications. Computed tomography (CT) and subsequent three-dimensional (3D) rendering has improved the surgeon's ability to visualise and classify intra-articular fractures, thereby influencing the decision to operate. Three-dimensional printing is a rapidly expanding technology and if applied as an adjuvant to CT, could add a tangible element to fracture assessment and classification.

The reliability of current classification systems of tibial plateau fractures has been assessed in a variety of publications. Taşkesen et al. ${ }^{1}$ assessed the intra- and inter-observer reliability of the Arbeitsgemeinschaft für Osteosynthesefragen-Orthopaedic Trauma Association (AO-OTA), Schatzker, Hohl and Moore, Luo and revised Duparc systems using plain (2D) radiography as well as CT. The inter-observer reliability of the Schatzker system was substantial when CT was used and moderate for plain radiographs. The AO-OTA classification, the Hohl and Moore, and the Luo classifications were all moderate for both CT and standard radiographs. The Duparc classification system was the least reliable. Mellema et al. $^{2}$ compared the inter-observer reliability of the Schatzker system with the Luo classification system using either 2D CT images or 2D as well as 3D CT images. Both systems showed only fair reliability, with the Schatzker classification being significantly better. However, the added use of 3D CT did not improve the reliability.

Maripuri et al. ${ }^{3}$ compared the interobserver reliability of the AO-OTA, Schatzker, and Hohl and Moore systems. Although the Schatzker system was better, none was ideal as the reliability ranged from poor to moderate. Millar et al. ${ }^{4}$ carried out a systematic review of tibial plateau fracture classification systems. They identified 38 systems, of which five were sub-classifications of existing systems, and only 11 systems associated fracture classification with clinical outcome. The review concluded that 2D and 3D CT improved interobserver reliability estimates, but that more detailed assessment was required to help predict outcomes and to guide clinical decisions.

A Medline search using the PubMed search engine using the search terms 'three dimensional/3D printing assisted tibial plateau fracture' revealed five studies, one of which was in Chinese, and none of which assessed the inter-observer reliability of tibial plateau fracture classification. Huang et al. ${ }^{5}$ showed that a $3 \mathrm{D}$-printed template improved the accuracy of plating and screwing for complicated tibial plateau fractures. Yang et al. ${ }^{6}$ assessed 3D model use in seven lateral tibial plateau malunions and concluded improved outcome. Lou et al. ${ }^{7}$ compared conventional tibial plateau surgery with surgery assisted by 3D-printing technology in 72 patients. The authors reported a decrease in intra-operative blood loss, operative time and fluoroscopy use in the 3D printing-assisted group. A systematic review ${ }^{8}$ of $3 \mathrm{D}$ printing in tibial plateau fracture management found shorter operative time, less intra-operative blood loss and faster union times when 3D model-assisted open reduction internal fixation (ORIF) was used compared with conventional ORIF. However, there was no significant difference in follow-up outcomes or complications.

In light of the fact that it is accepted that 3D CT is known to assist in surgical preparation and planning, it is not known whether these technologies can assist in reliably classifying tibial plateau fractures. The aim of this retrospective study was therefore to compare 3D models with 3D CT in terms of the reliability of classification of tibial plateau fractures using the Hohl and Moore and the Schatzker classification systems, and to establish whether 3D model use might influence surgical management in the future.

\section{Materials and method}

The sample comprised the $20 \mathrm{CT}$ scans of patients with tibial plateau fractures available in the radiology archive of Dr George Mukhari Academic Hospital, Ga-Rankuwa. Of the 27 knee CT scans available on the patient archive system, only 20 scans identified tibial plateau fractures. Thus, all the available data was used. This study did not involve patient contact and did not consider the injuries commonly associated with tibial plateau fractures (fracture dislocation, meniscal and multi-ligamentous injuries). No assumptions were made regarding patient demographics, mechanism of injury, or open or closed injuries. For the purpose of the study, soft tissue injuries were not considered, and all patients were presented as adults (fit for surgical management).

The CT data was processed digitally (Phillips IntelliSpace Portal system, Phillips, Netherlands) to produce 3D images to be assessed on a computer screen (Figure 1) and which could be rotated in all planes at the observer's discretion. Patient identifiers were replaced with research numbers. The volumetric data from the CT scans was converted to Standard Tessellation Language files used to print corresponding 3D models at a 1:1 scale by additive printing (Ultimaker II 3D printer, Ultimaker BV, Netherlands). The models used (both 3D CT and 3D-printed models) consisted of a distal femur and proximal tibia. The patella was removed as it has no bearing on classification. Polylactic acid was used as the medium because it is a rigid, bio-friendly plastic and is manufactured in a matt white colour resembling cortical bone (Figure 2). All models were printed using a $20 \mu \mathrm{m}$ definition setting in the same printer using the same batch of material. The cost of 3D model

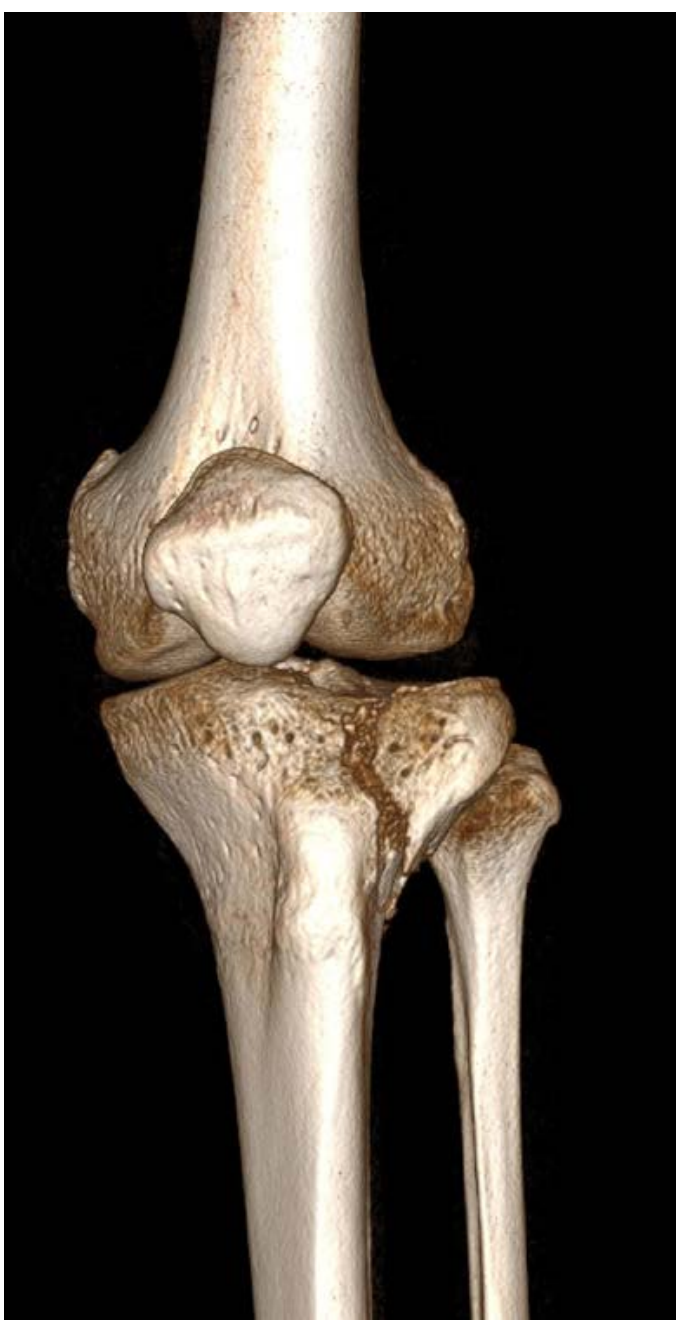

Figure 1. A screenshot of a digital 3D CT scan used 


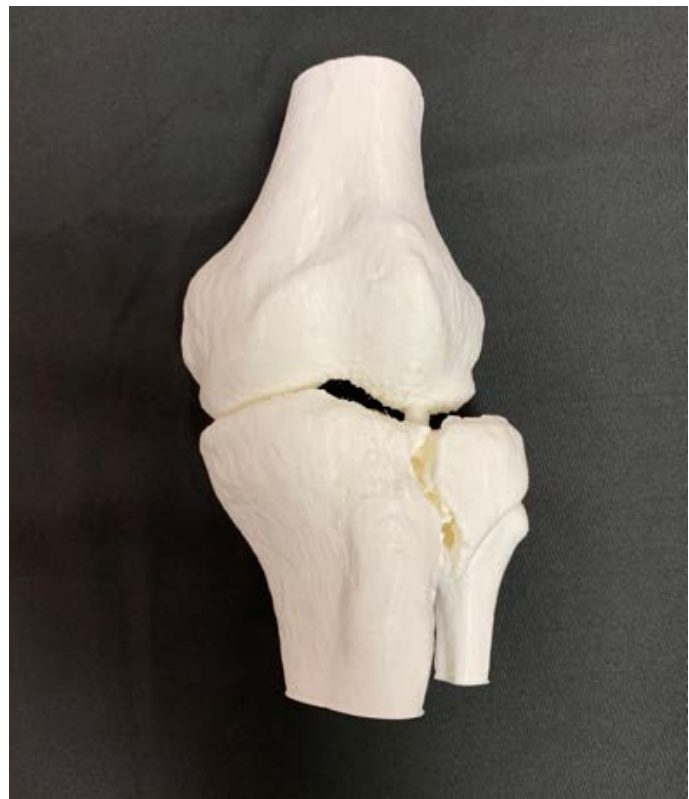

Figure 2. A picture of a 3D printed model used

manufacturing at the time of publication was estimated at R1 500 per model. Manufacturing time was estimated at roughly 24 hours, depending on the model size and complexity.

Standardised descriptive illustrated classification guides were used by all observers: these were of the Schatzker system types $\mathrm{I}-\mathrm{VI}$ as described by Zeltser and Leopold ${ }^{1}$ and the Hohl and Moore classification system types I-V. ${ }^{3}$ Six observers practising in the field of orthopaedic surgery volunteered to complete a series of questionnaires. The observers comprised two senior surgeons with more than ten years of experience (as a registered orthopaedic surgeon), two junior surgeons with less than ten years of experience (as a registered orthopaedic surgeon), and two registrars. All the observers were employed as full-time staff in an orthopaedic department. They completed the questionnaires individually in three different sessions. The 3D images were viewed on a computer screen and the 3D models were assessed by means of tactile examination and direct inspection.

In session one the observers had access to the 3D CT images only. In session two, several days later, depending on availability (estimated at a two-to-five day delay), the observers assessed only the 3D models. In the third session (estimated two-to-five day delay from session 2), the observers assessed the 3D CT images while handling and inspecting the corresponding 3D model, in order to assess aspects that would more directly influence treatment and management. As the aim of the study was to compare 3D models to $3 \mathrm{D} C T$, the observers did not have access to plain radiographs nor to 2D CT data.

The first and second session questionnaires required the observers to:

- Classify the specific tibial plateau fracture using the two classification systems if applicable according to the observer

- Choose between surgical and conservative management for each case, assuming the patient was healthy and fit for surgery
- If the management was to be surgical, specify the approach:

- Non-operative management

- Percutaneous screw fixation

- Circular external fixation

- Intramedullary nail

- Single plate and screw construct

- Multiple plate and screw constructs

- Assess whether it was possible to estimate how much bone graft would be required, as 'yes/no/maybe/not applicable'

For the third questionnaire the observers were asked their opinion on whether the 3D model was inferior, equal or superior to the 3D CT image with regard to the following characteristics: fracture description; fracture classification; decision-making; spatial awareness; and assessment of the intra-articular fracture pattern.

In addition, they were asked if they would consider printing a 3D model for future intra-articular fracture classification/decisionmaking, on a scale of yes/no/maybe.

The Kappa coefficient system was used to evaluate inter- and intra-observer reliability. Cohen's kappa was used for two raters and the Fleiss kappa (an adaptation of Cohen's kappa) for three or more raters. All statistical procedures were performed on IBM SPSS statistics 22 running on Microsoft Windows. The kappa interpretation is given in Table $I$.

\section{Results}

The inter-observer reliability kappa values for classifying the fractures are shown in Table II. There was fair agreement in the Hohl and Moore classifications and moderate agreement in the Schatzker classification system.

When assessing the classification systems by comparing the 3D CT image with the 3D model for each observer, an average value for all comparisons for all observers was obtained for each of the classification systems, and this revealed a moderate agreement and no difference between the classification systems (Table III).

When the observers assessed cases in terms of surgical vs conservative treatment using only the 3D CT images, only three out of 120 responses (six observers $\times 20$ cases) precluded conservative treatment, with all other responses being surgical. When assessing cases using the 3D models only, only one response precluded conservative management.

For the six possible surgical management options given to the observers, there was an overall difference in agreement between the decisions based on assessing only the 3D CT images compared with assessing only the 3D models, of $19 \%$ (23 of the possible

Table I: Kappa scale interpretation

\begin{tabular}{|l|l|}
\hline Kappa value & Interpretation \\
\hline$<0$ & Poor agreement \\
\hline $0.01-0.20$ & Slight agreement \\
\hline $0.21-0.40$ & Fair agreement \\
\hline $0.41-0.60$ & Moderate agreement \\
\hline $0.61-0.80$ & Substantial agreement \\
\hline $0.81-1.00$ & Almost perfect agreement \\
\hline
\end{tabular}

Table II: Kappa inter-observer values for all six observers

\begin{tabular}{|l|c|c|c|}
\hline \multicolumn{1}{|c|}{ System } & 3D CT image reliability kappa value & 3D-printed model reliability kappa value & Kappa interpretation \\
\hline Hohl and Moore & 0.319 & 0.318 & Fair agreement \\
\hline Schatzker & 0.57 & 0.48 & Moderate agreement \\
\hline
\end{tabular}


Table III: Average kappa values across all six observers when comparing each observer's scores for CT image classification compared with their score for CT model classification

\begin{tabular}{|l|c|l|}
\hline System & Kappa value & Kappa interpretation \\
\hline Hohl and Moore & 0.56 & Moderate agreement \\
\hline Schatzker & 0.52 & Moderate agreement \\
\hline
\end{tabular}

\section{3 differing proposed procedures}

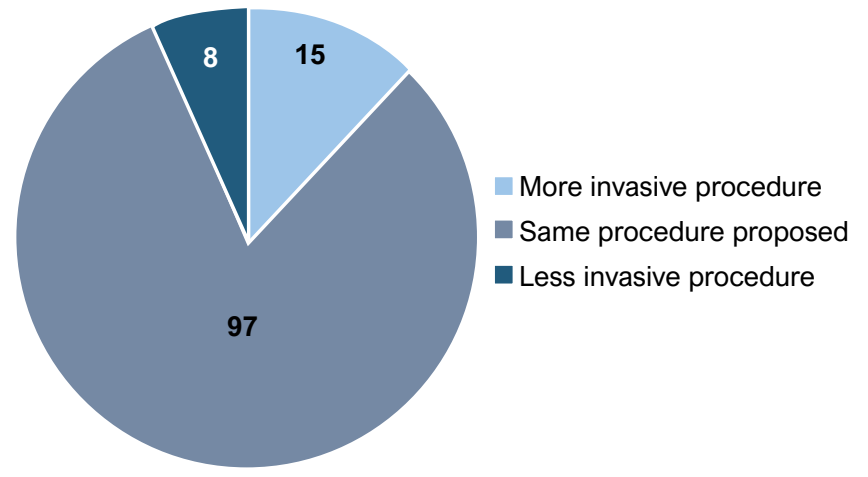

Figure 3. Effect on surgical management due to 3D model

120 procedures). In order to investigate whether the assessment of the models would result in a less, equal, or more invasive procedure, the procedures were scored on a scale of 1 to 6 , representing the six options given, with 1 being least, and 6 the most invasive procedure. Of the 23 differing procedures, 15 were more invasive (65\% of differing proposed procedure cases or $12.5 \%$ of the total), and eight were less invasive (35\% of differing proposes procedure cases or $6.7 \%$ of the total) (Figure 3 ).

The ability to assess the quantity of bone graft required was assessed on a yes/no/maybe/not applicable scale. The 'yes' category was scored more frequently when assessing the 3D models ( $75 \%$ vs $64 \%)$ (Table IV).

The observers' opinions on whether the 3D model was of inferior, equal or superior value in assessing the various characteristics are shown in Table $V$.

Finally, the observers were asked if they would consider printing a 3D model in the future and their responses were positive, based on case-specific needs. Those mentioned were comminuted intraarticular fractures; severely displaced fractures; bony defects; and Schatzker types IV-VI.

\section{Discussion}

Three-dimensional models have been described as useful in preoperative planning ${ }^{9}$ and the effect of model use on patient outcome has been investigated; ${ }^{7}$ however, no publications were found on the influence of 3D models on patient management or on the interobserver reliability in tibial plateau fracture classification regardless of the classification system used.

Of the 38 classification systems used to classify tibial plateau fractures, none appears to be ideal. ${ }^{3}$ For example the Schatzker classification system was found to be more reliable when 3D CT was used to assess tibial plateau fractures compared with plain radiography in one study ${ }^{1}$ but not in another. ${ }^{2}$ In this study, the Schatzker system (moderate reliability) was superior to the Hohl and Moore system (fair reliability) which was in agreement with Taşkesen et al. ${ }^{1}$

When used for acetabular fracture classification, the use of 3D models has been reported to have improved inter-observer reliability
Table IV: Observers' opinions on whether it was possible to assess the quantity of bone graft required

\begin{tabular}{|l|c|c|c|c|}
\hline \multirow{2}{*}{ Opinion } & \multicolumn{2}{|c|}{ 3D CT image } & \multicolumn{2}{c|}{ 3D model } \\
\cline { 2 - 5 } & No. & $\%$ & No. & $\%$ \\
\hline Yes & 64 & 53 & 75 & 63 \\
\hline No & 32 & 27 & 23 & 19 \\
\hline Maybe & 17 & 14 & 19 & 16 \\
\hline Not applicable & 7 & 6 & 3 & 3 \\
\hline
\end{tabular}

Table V: Observers' opinions on whether the 3D model was of inferior, equal or superior value

\begin{tabular}{|l|c|c|c|c|c|c|}
\hline \multirow{2}{*}{ Feature } & \multicolumn{2}{|c|}{ Inferior } & \multicolumn{2}{c|}{ Equal } & \multicolumn{2}{c|}{ Superior } \\
\cline { 2 - 7 } & No. & $\%$ & No. & $\%$ & No. & $\%$ \\
\hline Fracture description & 19 & 16 & 61 & 51 & 40 & 33 \\
\hline Fracture classification & 8 & 7 & 90 & 75 & 22 & 18 \\
\hline Decision-making & 9 & 18 & 65 & 54 & 46 & 38 \\
\hline Spatial awareness & 9 & 8 & 20 & 17 & 91 & 76 \\
\hline $\begin{array}{l}\text { Intra-articular fracture } \\
\text { pattern assessment }\end{array}$ & 7 & 6 & 33 & 28 & 80 & 67 \\
\hline
\end{tabular}

compared with 3D CT. ${ }^{10}$ In this study, however, comparison of these 3D tools (CT and models) showed no statistically significant benefit in inter-observer reliability with $3 \mathrm{D}$ model use irrespective of the classification system used. This should imply that a more reliable approach to tibial plateau fracture classification remains elusive and the addition of 3D models did not replicate the success reported in acetabular fractures. ${ }^{10}$

The application of 3D models in the medical field has expanded dramatically in the last five years. The benefit of their use in orthopaedic pre-operative planning is frequently reported but poorly defined. ${ }^{9}$ The influence of 3D models on decision-making remains broadly untested in terms of surgical or conservative management. In this study, the observer responses indicated that $1 \%$ more cases would have received surgical management when the assessment was based on the 3D model. In addition, the theoretical proposed procedure based on 3D model assessment differed from the proposed procedure based on 3D CT assessment in $19 \%$ of cases, implying that the use of 3D models should be seen as an adjunct to planning and should not be used alone. This could alter management in nearly one out of every five tibial plateau patients managed surgically. The use of 3D models alone would have resulted in a theoretically more invasive procedure in $12.5 \%$ of patients and a theoretically less invasive procedure in $6.5 \%$ of patients. Although 3D models changed the specific proposed procedure in $19 \%$ of cases, the change was in a relatively neutral direction, meaning (based on a scoring system) it was neither more nor less invasive. A recent literature review ${ }^{11}$ on the use of $3 \mathrm{D}$ models in the medical field reported several advantages but no definitive conclusions on the influence on surgical outcomes. It did, though, point out the need for a formal cost-effectiveness analysis.

Various publications ${ }^{7,8,12}$ have reported decreased intra-operative blood loss and shorter operative time associated with 3D model use, but lack explanations as to the root of the benefit and there is no evidence to support improvements in outcome. This study found 3D models to be superior to 3D CT imaging in terms of spatial awareness and specifically the observer's ability to assess intraarticular fracture patterns. Although these are promising features, the effect on patient outcomes needs more study. It should also 
be noted that publications ${ }^{7}$ reporting on decreased operative time using 3D models also reported increased time spent on preoperative planning.

Although the observers in this study assessed 3D models as being superior to $3 \mathrm{D} \mathrm{CT}$ when considering the ability to estimate the quantity of bone graft required, this could not be confirmed intra-operatively.

If the use of $3 \mathrm{D}$ models is considered a valuable adjunct to treatment, the cost must also be considered. Cost assessment should include the CT scan, model manufacturing consumables, printing time, as well as time spent on pre-operative surgical simulation. A broader consideration of the true cost of 3D models may have a negative impact on their use.

The limitations of this study include a fairly small population group. As the patient archive system was manually stored off site only a limited number of CT scans were available. The use of a basic fundamental imaging modality (radiographs) was excluded in order to compare novel strategies. The observers did not have access to 2D CT images. Due to the various observers' obligations to patient care, the timing of questionnaire sessions was not strictly standardised. The study considers only tibial plateau fractures and does not consider associated soft tissue injuries. The study involves retrospective analysis of patient data and therefore no conclusion can be drawn relating to patient outcome.

\section{Conclusions}

Inter-observer reliability was not significantly different when assessing the Schatzker or Hohl and Moore classification systems using either the 3D models or the 3D CT images. The assessment of the $3 \mathrm{D}$ models did not favour surgical or conservative treatment significantly but did alter the observers' proposed surgical procedures in $19 \%$ of cases Observers reported 3D models equal to 3D CT imaging in terms of fracture description, fracture classification and decision-making. Model use was superior to 3D CT in terms of spatial awareness and intra-articular fracture description.

The use of $3 \mathrm{D}$ models in tibial plateau fracture pre-operative planning needs further study. The clinical impact of 3D model use (including cost, manufacturing time and radiation exposure) should be weighed against the potential benefits.

\section{Acknowledgements}

The author would like to thank Dr S Matshidza and Dr E D'alton for their support and guidance in carrying out this research, which was carried out in partial fulfilment for the degree MMed.

Supervisor: Dr Steven Matshidza, Consultant and Head of Clinical Department, Orthopaedics UFS/ Universitas Hospital, Bloemfontein

Co-supervisor: Dr Eduard Johan D'alton, Dr George Muhkari Hospital, Sefako Makgatho Health Sciences University, Ga-Rankuwa, Pretoria

\section{Ethics statement}

The author declares that this submission is in accordance with the principles laid down by the Responsible Research Publication Position Statements as developed at the $2 \mathrm{nd}$ World Conference on Research Integrity in Singapore, 2010. Prior to commencement of the study, ethical approval was obtained from the university's research ethics committee (approval number SMUREC/M/258/2015: PG).

\section{Declaration}

The authors declare authorship of this article and that they have followed sound scientific research practice. This research is original and does not transgress plagiarism policies.

\section{ORCID}

Joubert JA (D) https://orcid.org/0000-0001-6502-9806

\section{References}

1. Taşkesen A, Demirkale I, Okkaoğlu MC, et al. Intra-observer and inter-observer reliability assessment of tibial plateau fracture classification systems. Eklem Hastalik Cerrahisi. 2017;28:177-81. doi: 10.5606/ehc.2017.56816.

2. Mellema JJ, Doornberg JN, Molenaars RJ, et al. Inter-observer reliability of the Schatzker and Luo classification systems for tibial plateau fractures. Injury. 2016;47:944-49. doi: 10.1016/j. injury.2015.12.022.

3. Maripuri SN, Rao P, Manoj-Thomas A, Mohanty K. The classification systems for tibial plateau fractures: how reliable are they? Injury. 2008;39:1216-21. doi: 10.1016/j.injury.2008.01.023.

4. Millar SC, Arnold JB, Thewlis D, Fraysse F, Solomon LB. A systematic literature review of tibial plateau fractures: What classifications are used and how reliable and useful are they? Injury. 2018;49:473-90. doi: 10.1016/j.injury.2018.01.025

5. Huang $\mathrm{H}$, Hsieh MF, Zhang $\mathrm{G}$ et al. Improved accuracy of 3D-printed navigational template during complicated tibial plateau fracture surgery. Australas Phys Eng Sci Med. 2015;38:109-17. doi: 10.1007/s13246-015-0330-0.

6. Yang P, Du D, Zhou Z, et al. 3D printing-assisted osteotomy treatment for the malunion of lateral tibial plateau fracture. Injury. 2016;47:2816-21. doi: 10.1016/j.injury.2016.09.025

7. Lou Y, Cai L, Wang C, et al. Comparison of traditional surgery and surgery assisted by three dimensional printing technology in the treatment of tibial plateau fractures. Int Orthop. 2017;41:1875-80. doi: 10.1007/s00264-017-3445-y.

8. Xie L, Chen C, Zhang Y, et al. Three-dimensional printing assisted ORIF versus conventional ORIF for tibial plateau fractures: A systematic review and meta-analysis. Int J Surg. 2018;57:35-44. doi: 10.1016/j.ijsu.2018.07.012.

9. Bizzotto N, Sandri A, Regis D, et al. Three-dimensional printing of bone fractures: a new tangible realistic way for preoperative planning and education. Surg Innov. 2015;22:548-51. doi: 10.1177/1553350614547773.

10. Hurson C, Tansey A, O'Donnchadha B, et al. Rapid prototyping in the assessment, classification and preoperative planning of acetabular fractures. Injury. 2007;38:1158-62. Epub 2007 Sep 19.

11. Tack P, Victor J, Gemmel P, Annemans L. 3D-printing techniques in a medical setting: a systematic literature review. Biomed Eng Online. 2016;15:115-36. doi: 10.1186/s12938-016-0236-4.

12. Giannetti S, Bizzotto N, Stancati A, Santucci A. Minimally invasive fixation in tibial plateau fractures using an pre-operative and intra-operative real size 3D printing. Injury. 2017;48:784-88. doi: 10.1016/j.injury.2016.11.015. 